\title{
Comparison of Macro-and Micro-porosity of a Titanium Mesh for Guided Bone Regeneration: An In Vivo Experimental Study
}

\author{
MOTOKI SENOO ${ }^{1,2}$, AKIRA HASUIKE ${ }^{2,3}$, TAKANOBU YAMAMOTO ${ }^{2}$, YASUMASA OZAWA ${ }^{2}$, \\ NORIHISA WATANABE ${ }^{2}$, MITSUAKI FURUHATA ${ }^{1,2}$ and SHUICHI SATO ${ }^{2,3}$ \\ ${ }^{1}$ Division of Applied Oral Sciences, Nihon University Graduate School of Dentistry, Tokyo, Japan; \\ ${ }^{2}$ Department of Periodontology, Nihon University School of Dentistry, Tokyo, Japan; \\ ${ }^{3}$ Dental Research Center, Nihon University School of Dentistry, Tokyo, Japan
}

\begin{abstract}
Background/Aim: Guided bone regeneration $(G B R)$ is one of the surgical methods used for vertical ridge augmentation prior to dental implant placements. Titanium meshes have been used for osteogenic space maintenance in GBR sites by clinicians. We aimed to compare the influence of micropores and macropores in a titanium mesh on bone regeneration in a rat calvarial vertical GBR model. Materials and Methods: The calvaria of nine rats were exposed, and plastic cylinders were set bilaterally. Eighteen surgical sites were randomly allocated into three groups according to the materials of titanium lid and bone substitutes: microporous titanium lid+deproteinized bovine bone mineral (DBBM), macroporous titanium lid $+D B B M$, microporous titanium lid+carbonate apatite. Newly generated bone inside the cylinders was evaluated using micro-computed tomography (micro-CT). Furthermore, bone regeneration and angiogenesis were evaluated histologically at 12 weeks. Results: Quantitative volumetric analyses using micro-CT showed a gradual increase in bone volume inside the cylinders in all three groups. Histological observation confirmed vigorous bone regeneration in the microporous groups compared to that in the macroporous group. In the upper part of the cylinders, soft tissue invaded the GBR site by passing through the pores of the macroporous mesh. The blood vessels in the upper part of the cylinders were smaller in the microporous groups than in the macroporous group. There was no difference in bone formation between cylinders
\end{abstract}

This article is freely accessible online.

Correspondence to: Akira Hasuike, Department of Periodontology, Nihon University School of Dentistry, 1-8-13 Kanda-Surugadai, Chiyoda-ku, Tokyo 101-8310, Japan. Tel: +81 332198349, e-mail: hasuike.akira@nihon-u.ac.jp

Key Words: Alveolar ridge augmentation, bone regeneration, dental implants, skull, X-ray microtomography, GBR. filled with DBBM or carbonate apatite. Conclusion: Microvasculature penetrates 50- 4 m diameter micropores and accelerates bone formation inside the cylinder, which was set on rat calvaria. The microporous titanium mesh can facilitate angiogenesis from both the dura mater and periosteal in vertical ridge augmentation. Our data showed superiority of microporous titanium vascular permeability and osteoconductivity, supporting bone growth.

The volumetric change in hard tissue dimensions is a critical consequence of tooth loss, which limits the optimal placement of dental implants. Many therapeutic modalities have been suggested to enhance the reconstruction of deficient alveolar ridges prior to implant placement (1). Guided bone regeneration (GBR) is the most commonly used method for vertical ridge augmentation (VRA); it enhances bone volume by guiding osteogenic cells to an area beyond the original skeletal envelope (2). This surgical technique is based on the principle that mechanical barriers hinder epithelial and fibrous tissue invasion into the augmented bone. VRA is biologically demanding, as the influx of stem cells and blood flow needs to reach a certain distance from the existing bone for new bone formation. To explore these biological events, it is crucial to conduct in vivo assessments of GBR using preclinical models. A well-standardized rat GBR model with plastic cylinders placed on calvarial defects was developed by our group (3-6).

GBR can be performed using several techniques, which are mainly based on the selection of bone substitutes and mechanical barriers. So far, clinicians select techniques, according to their experience and preference rather than biological backgrounds. Several kinds of membranes have been used as mechanical barriers to prevent the invasion of non-osteogenic fibrous tissue in regenerating bone defects. Conventionally, non-resorbable barrier membranes made of a polymer material such as polytetrafluoroethylene (PTFE) have been used since a long time (7-9). Resorbable barrier membranes made of collagen or polymer materials are also 
being used in clinical settings (8-10). Although these commercially available membranes were reported to be highly predictable, they have several limitations. The first is their susceptibility to bacterial contamination. Conventional membranes have a porous body or fibers, and the matrix has an abundance of intricate small cavities. Thus, following membrane exposure, bacteria may invade the small cavities and cause a local infection. Second, membranes have a poor performance in terms of space maintenance, which is a fundamental prerequisite for any bone regeneration procedure. Unfortunately, the stiffness of membranes can be compromised by the pressure induced by the soft tissue inflammatory reaction and chewing process. To prevent the membranes from collapsing, the membrane should be thick so that the space for osseous regeneration becomes narrow.

Recently, titanium products have been used to compensate for the limitations of these conventional membranes $(8,9)$. Titanium-reinforced PTFE membranes are among the most documented membranes in VRA. Previous studies showed successful histological bone formation by VRA with a titanium-reinforced PTFE membrane and an average bone gain of $5.45 \mathrm{~mm}$ in bone height (11). Although this material possesses characteristics such as biocompatibility, smoothness, and stiffness, the adaptation and fixation with bone pins or screws to the recipient site are technically demanding and time-consuming. Titanium meshes have also been used by clinicians for VRA since a long time (12). According to current clinical reports, VRA with a titanium mesh has strong osteogenesis predictability, and most researchers achieved an average gain of 5-7 $\mathrm{mm}$ in bone height (13). Titanium meshes in the dental market are macroporous, as the pore diameter is in the millimeter range. Since titanium mesh is far from occlusive, there is a possibility that fibrous tissue could intrude into the bone defect area and jeopardize bone regeneration. The influence of macroporosity remains controversial $(6,14,15)$. Several researchers have suggested that the pores of a titanium mesh play an essential role in establishing the supplement of osteogenic cells, growth factors, and blood supply from the periosteal overlying the barrier controversial $(6,14)$.

Recently, Hasegawa et al. developed a microporous titanium mesh with a pore diameter of $20 \mu \mathrm{m}$ and a center-tocenter distance of $50 \mu \mathrm{m}$ (16). The use of a laser processing system made it possible to create such tiny perforations at a high density. They reported strong osteogenesis predictability for internal GBR when this microporous titanium mesh was used with an autogenous iliac bone graft in a box-shaped ridge defect model of the dog mandible (17). However, as the defect is well contained, this internal box-shaped ridge defect is far from the clinical reality of VRA. Thus, it is necessary to conduct preclinical studies that reflect the clinical usage of VRA. In 2017, a microporous titanium mesh (Ti honeycomb
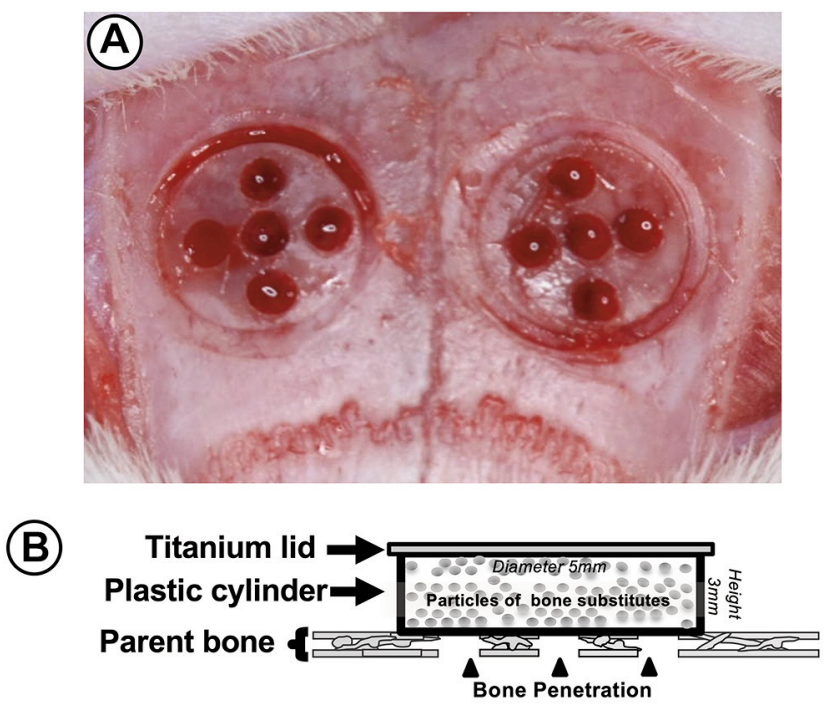

Figure 1. A circular groove was made with a trephine bur, and five small holes were drilled with a round bur to allow for marrow penetration (A); A schematic depiction of the rat experimental model (B).

membrane) developed by Hasegawa et al. was released in the Japanese market. Recently, Ishikawa et al. reported the first case of VRA with a microporous titanium mesh (18). They reported successful results of simultaneous GBR with implant placement in the maxillary anterior region. A vertical bone gain of 2-3 mm was observed 6 months after VRA, which could support the esthetic buccal implant papillae. Although the authors attributed the success of the procedure to the low elasticity and high formability of the product, the effect of microporosity was not elucidated in this study. Therefore, the present study aimed to compare the influence of micropores and macropores in titanium mesh used with bone substitutes on bone regeneration in a rat calvarial vertical GBR model. The dynamics of osteogenesis were elucidated by microcomputed tomography (micro-CT) and histological examination, and angiogenesis was also elucidated by histological examination.

\section{Materials and Methods}

Animals. Eighteen surgical sites (250-300 g) of nine-week-old Fischer male rats were used in the experiments. Our primary outcome was percentage of newly formed bone in histology. The sample size was calculated with $\mathrm{G}^{*}$ Power software v. 3.1(University of Dusseldorf, Dusseldorf, Germany) using Wilcoxon-MannWhitney test at an alpha level of 0.05 and a statistical power of $95 \%$ based on the previous study (6), in which percentage of newly formed bone from occlusive and non-occlusive barriers showed significant statistical difference between $25 \pm 3 \%$ and $17 \pm 3 \%$. All animals were housed in cages to ensure standardized environmental factors during the experimental period. 
(A)

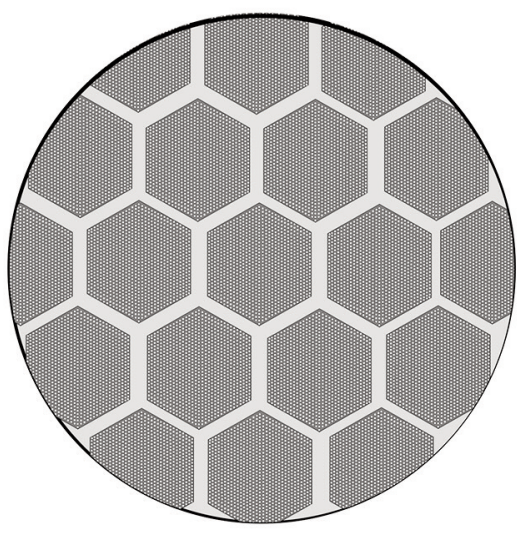

(C)

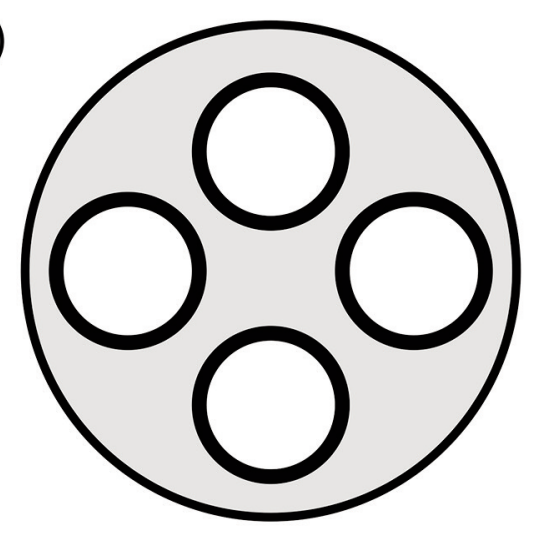

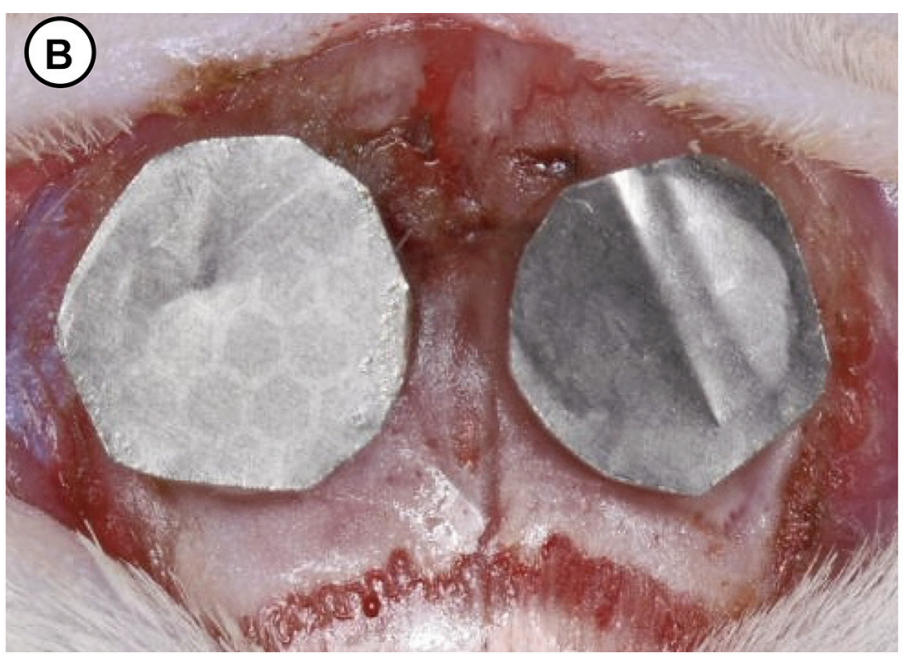

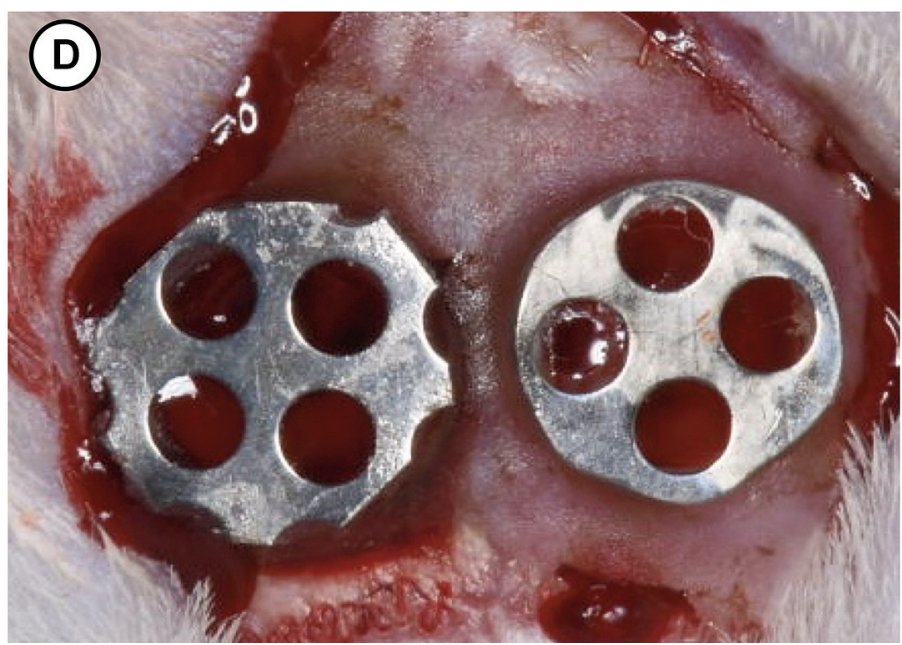

Figure 2. A schematic depiction of microporous titanium lids (A); cylinders with microporous titanium lids were set on the both sides of calvaria (B); a schematic depiction of macroporous titanium lids (C); cylinders with macroporous titanium lids were set on both sides of calvaria (D).

Surgical procedures. The standardized rat calvarial vertical GBR model developed by the present group was used (3-6). Before the surgical session, the animals were anesthetized via initial inhalation of $4 \%(\mathrm{v} / \mathrm{v})$ isoflurane for 2 minutes, followed by intraperitoneal injection of a mixture of $0.15 \mathrm{mg} / \mathrm{kg}$ dexmedetomidine hydrochloride, $2.0 \mathrm{mg} / \mathrm{kg}$ midazolam, and $2.5 \mathrm{mg} / \mathrm{kg}$ butorphanol tartrate. A $0.5 \mathrm{ml}$ solution of 1:80,000 dilution of lidocaine (Xylocaine; Astra Zeneca, Osaka, Japan) was administered locally to control bleeding and pain. After shaving and disinfecting with $70 \%$ ethanol swabs in the region between the eyes and the posterior end of the skull, a midline incision of $6.0 \mathrm{~cm}$ was made, and a mucoperiosteal flap was elevated with a small sharp periosteal elevator to expose the cranial vertex. Two standardized circular grooves of 5-mm-diameter were made at the center of each parietal bone using a trephine bur with an external diameter and a trephine drill. The trephine burr was used under constant irrigation with $0.9 \%$ saline to prepare the grooves without damaging the inner dura of the cranial bone. To induce bleeding, five small penetration holes were drilled with a small round burr (Figure 1A). After exhaustively washing the surgical area with sterile saline to remove any bone scraps, plastic cylinders $(5.0 \mathrm{~mm}$ diameter, $3.0 \mathrm{~mm}$ height) were pressed into the circular grooves (Figure 1B). The plastic cylinders were filled with bone substitutes and covered with titanium lids. Using a computer-generated random sequence, eighteen surgical sites were randomly allocated into three groups according to the titanium lid and bone substitute materials:

1. Microporous titanium lid (Ti honeycomb membrane, Morita, Tokyo, Japan) (Figure 2A and B)+deproteinized bovine bone mineral (DBBM, Bio-Oss ${ }^{\circledR}$, Geistlich-Pharma, Wolhusen, Switzerland): MicroTi+ DBBM.

2. Macroporous titanium lid (titanium mesh, Jeil Medical, Seoul, Republic of Korea) (Figure 2C and D)+DBBM (Bio-Oss ${ }^{\circledR}$, Geistlich-Pharma, Wolhusen, Switzerland): MacroTi+DBBM.

3. Microporous titanium lid (Ti honeycomb membrane, Morita, Tokyo, Japan) (Figure $2 \mathrm{~A}$ and $\mathrm{B})+$ carbonate apatite $\left(\mathrm{CO}_{3} \mathrm{AP}\right.$, Cytrans ${ }^{\circledR}, \mathrm{GC}$, Tokyo, Japan): MicroTi+ $\mathrm{CO}_{3} \mathrm{AP}$. 


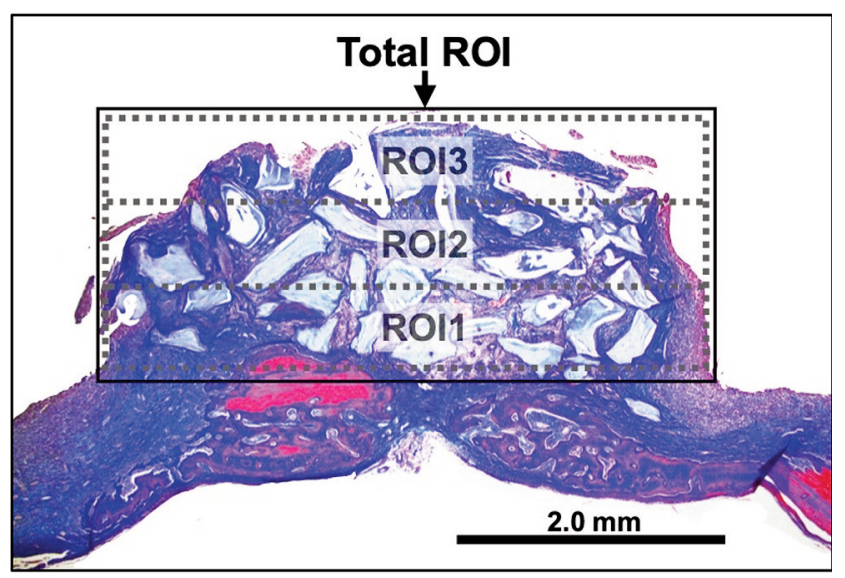

Figure 3. Image representing regions of interest (ROI) in histological assessment.

After surgery, the skin and periosteum were repositioned and sutured with simple resorbable interrupted sutures (VSORB 4-0, Washiesu Medical, Tokyo, Japan). The day of surgery was designated as day 0 . The rats were euthanized 12 weeks postsurgery.

Micro CT analysis. The total volume of the minerals at the surgical sites was evaluated repeatedly by radiography. An in vivo micro-CT system (R_mCT2 system; Rigaku, Tokyo, Japan) was used for imaging at $0,4,8$, and 12 weeks postoperatively without euthanasia. The rats were seated on an imaging stage and anesthetized by inhalation of an oxygen-isoflurane mixture via a facemask. The exposure parameters were $90 \mathrm{kV}$ and $100 \AA$, respectively. The region of interest (ROI) for micro-CT assessment was defined as the circular grooves made on the calvaria, each of which had a diameter of $5.0 \mathrm{~mm}$ and a height of $3.0 \mathrm{~mm}$. Images were reconstructed on a personal computer using the i-View software (i-View Image Center, Tokyo, Japan). The bone volume within the plastic caps in the voxel images was examined using the same software (i-View Image Center, Tokyo, Japan). The enhanced volume was calculated by subtracting the volume on day 0 from the values obtained from each follow-up. Measurements were taken by one experienced and blinded examiner (Y.O.) for micro-CT assessment.

Histological analysis. Histomorphometric analyses of histological sections, osteogenesis, and angiogenesis at the experimental GBR sites were conducted. All rats were euthanized by excess $\mathrm{CO}_{2}$ gas inhalation at 12 weeks postoperatively. The bone segments containing the cylinders were resected from the surgical sites and fixed in $10 \%$ neutral-buffered formalin, dehydrated, and embedded in paraffin wax blocks. Blocks were processed into 5 - $\mu \mathrm{m}$ sections at the central region of the cylinder and stained with Masson's trichrome staining to detect collagen and lamellar bone formation. Histomorphometric assessments of the sections were conducted under a light microscope and an image analyzer computer system using ImageJ software (National Institutes of Health, MD, USA). The total ROI for histomorphometric analysis was divided into

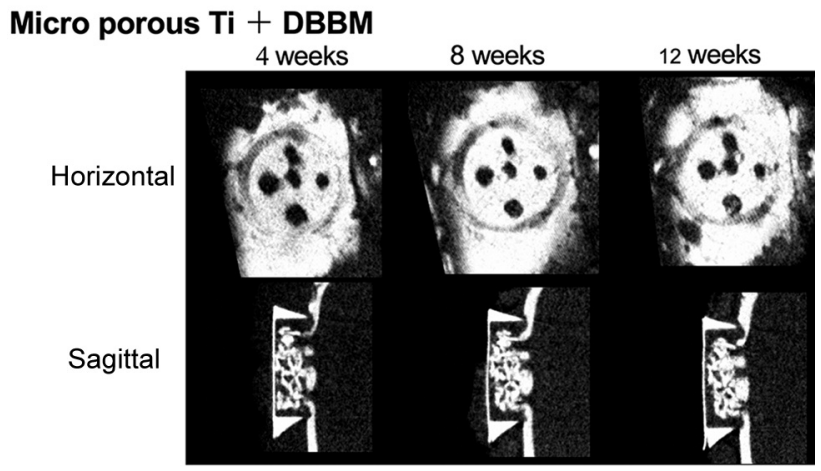

Ti mesh + DBBM

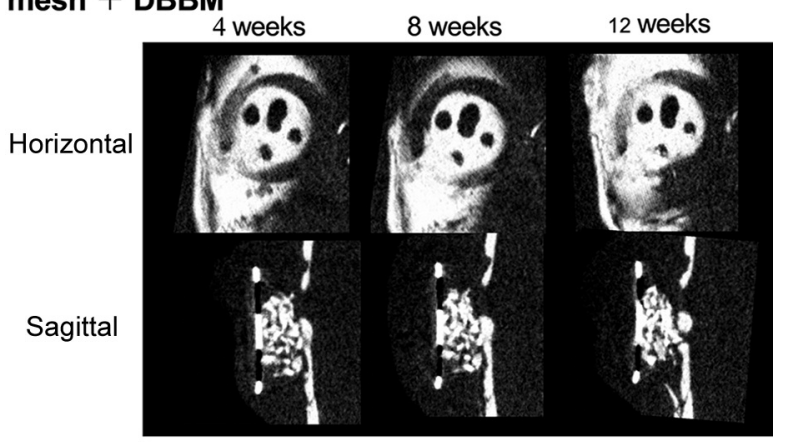

Micro porous $\mathrm{Ti}+\mathrm{CO}_{3} \mathrm{AP}$

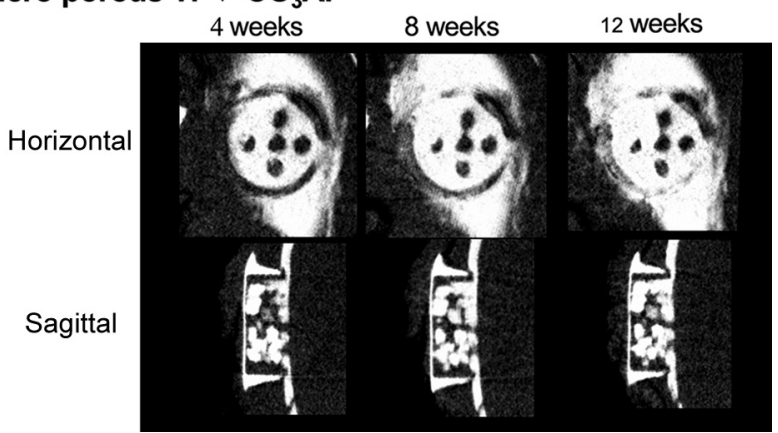

Figure 4. Micro-Computed tomography analysis of microporous titanium lids.

three parts: ROI1 referred to the region that covered $1.0 \mathrm{~mm}$ of the defect closest to the calvaria, ROI3 referred to the upper $1.0 \mathrm{~mm}$ of the defect, and ROI2 referred to the central region between ROI1 and ROI3 (Figure 3).

The following variables were assessed:

- Percentage of newly formed bone in total ROI: Area of newly formed mineralized bone tissue $\left(\mathrm{mm}^{2}\right) /$ area of total ROI $\left(\mathrm{mm}^{2}\right)$.

- Percentage of residual granules in the total ROI: area of residual granules $\left(\mathrm{mm}^{2}\right) /$ area of total ROI $\left(\mathrm{mm}^{2}\right)$.

- Number of blood vessels in each ROI: Vessels were quantified after scanning the section at $10 \times$ magnification. For the selection of vessels, only those showing lumens were quantified.

- Blood vessel size in each RIO: area of blood vessels $\left(\mathrm{mm}^{2}\right) /$ number of blood vessels in each ROI. 

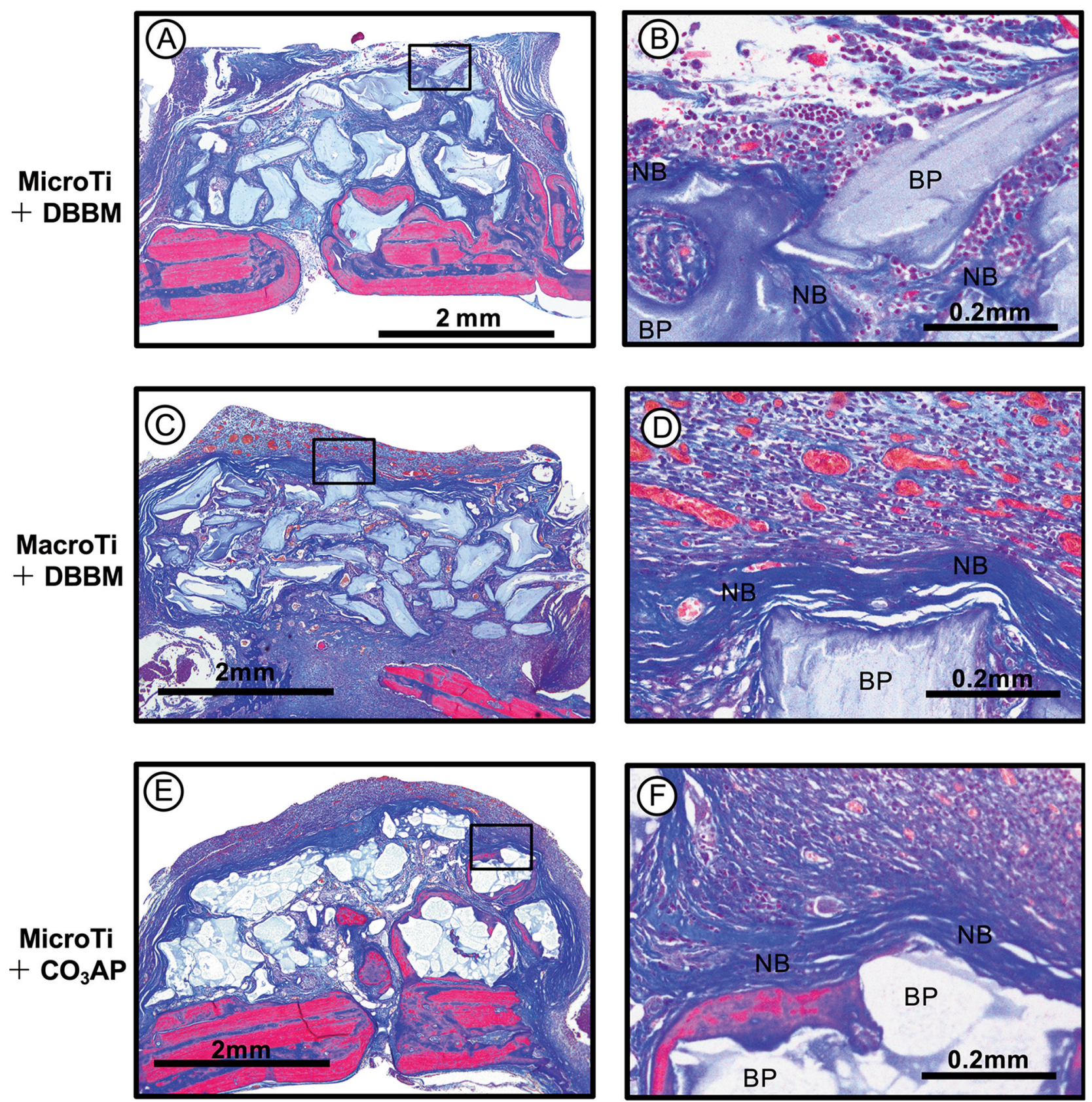

Figure 5. Representative histological specimens of each group at 12 weeks stained with Masson trichrome. Lower magnification (A, C, D); Higher magnification $(B, D, F)$. NB: New bone; BP: Biomaterial particle.

Measurements were taken by one experienced and blinded examiner (T.Y.) for histomorphometric assessment.

Statistical analysis. Means and standard deviations (SD) of each variable were collected and analyzed using EZR (Saitama Medical Center, Jichi Medical University, Saitama, Japan), a graphical user interface for R 2.13.0, (R Foundation for Statistical Computing, Vienna, Austria). Data were analyzed for statistical significance using a Kruskal-Wallis test with a Steel-Dwass post hoc test. $p<0.05$, were considered statistically significant.

\section{Results}

No surgical complications occurred during the cylinder placement. All animals healed normally, with no postoperative complications such as pain, fever, or infection.

Micro CT analysis. Micro-CT images of representative surgical sites in each group are shown in Figure 4. Although 
A

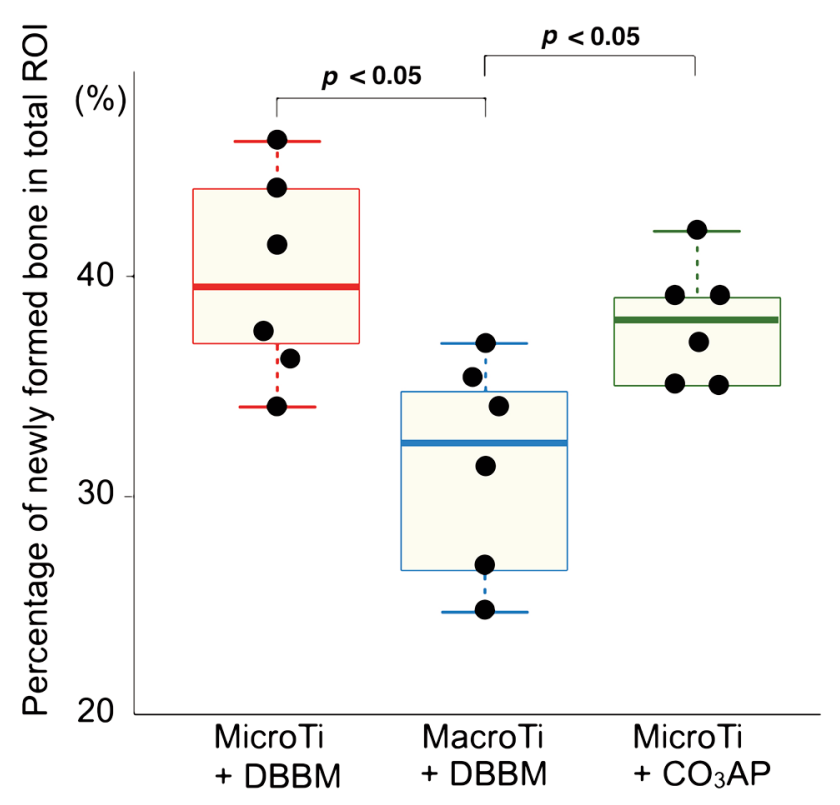

B

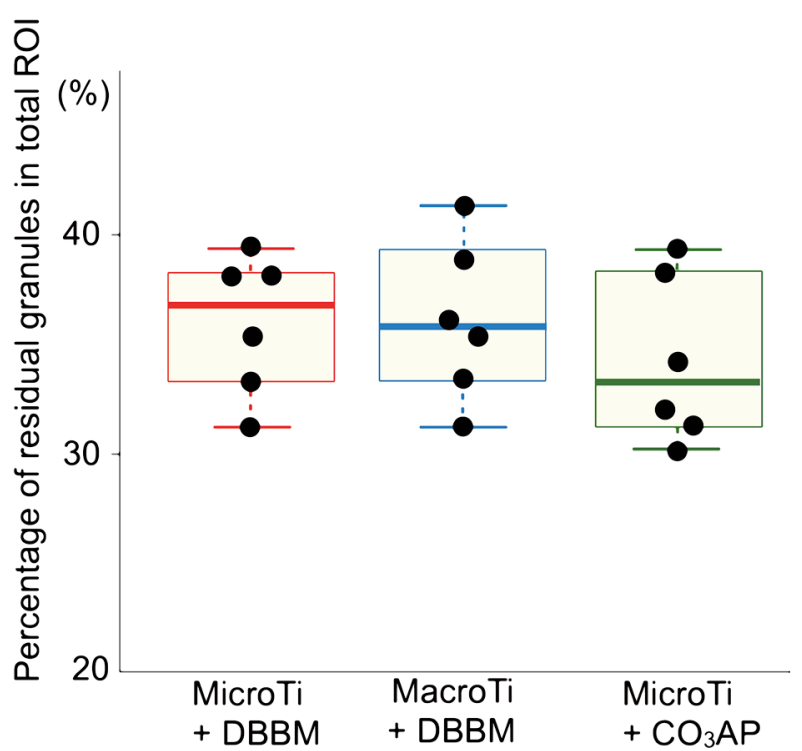

Figure 6. Histomorphometric measurements of each parameter after 12 weeks of healing are represented as box plots. Percentage of newly formed bone in total region of interest (ROI) (\%) (A); Percentage of residual granules in total ROI (\%) (B). The points show the data for all specimens. The median, percentiles, and maximum and minimum values of each group are displayed.

the pores of the macroporous titanium lid were confirmed by sagittal CT sections, the pores of the microporous titanium lid were not. Three-dimensional images obtained from micro-CT showed that the radiopaque contrast increased gradually inside the cylinders of all three groups. In the sagittal images of all three groups at 12 weeks, the gap between particles was partially filled with radiopacity. At all observational periods, the profile of each DBBM particle was confirmed clearly, in contrast to that of $\mathrm{CO}_{3} \mathrm{AP}$, which was shown as a mass of several particles. At 12 weeks, partial reossification images inside five small penetration holes were confirmed in all three groups. Quantitative volumetric analyses showed a gradual increase in the bone volume inside the cylinders in all three groups. The differences between the three groups were not statistically significant (Table I).

Histological analysis. Representative histological specimens stained with Masson's trichrome stain are shown in Figure 5 . The regenerated bone presented with a mature mineralized trabecular appearance. Osteoid borders on the bone surface indicate active osteogenesis. Soft tissue invasion was observed in all groups, and the most prominent growth of invasive soft tissue was observed in cylinders covered with macroporous titanium mesh. High magnification images of the area with soft tissue invasion, residual particle, and the newly formed bone tissue are presented in Figure 5B, D, and
Table I. Volumetric measurements from micro-computed tomography analysis $\left(\mathrm{mm}^{3}\right)$.

\begin{tabular}{lcccccc}
\hline $\begin{array}{l}\text { Bone volume } \\
\left(\mathrm{mm}^{3}\right)\end{array}$ & 4 weeks & \multicolumn{2}{c}{8 weeks } & \multicolumn{2}{c}{12 weeks } \\
\hline MicroTi+DBBM & 0.64 & $(2.09)$ & 1.17 & $(1.72)$ & 1.81 & $(1.50)$ \\
MacroTi+DBBM & 3.17 & $(0.54)$ & 3.42 & $(0.73)$ & 3.65 & $(0.28)$ \\
MicroTi+CO 3 AP & 2.04 & $(0.92)$ & 2.08 & $(1.46)$ & 2.54 & $(1.40)$ \\
\hline
\end{tabular}

Values are represented as mean (SD). There are no significant differences between any groups. MicroTi, Microporous titanium lid; MacroTi, macroporous titanium lid; DBBM, deproteinized bovine bone mineral; $\mathrm{CO}_{3} \mathrm{AP}$, carbonate apatite.

F. Smaller blood vessels were seen in cylinders covered with microporous titanium lids compared to that seen in cylinders with macroporous titanium lids.

The results of the quantitative analysis of histology are shown as box plots (Figure 6 and Figure 7). The percentage of newly formed bone tissue in cylinders covered with microporous titanium lids was significantly higher than that in cylinders with macroporous titanium lids (MicroTi+DBBM: $40.0 \pm 4.5 \%$, MicroTi+CO 3 AP: $37.8 \pm 3.6 \%$, Macro Ti +DBBM: $31.5 \pm 4.7 \%$ ) (Figure 6A). At 12 weeks, more than one-third of the bone substitute particles remained unabsorbed in all three groups (MicroTi+DBBM: 35.7×3.2\%, Macro Ti +DBBM: 


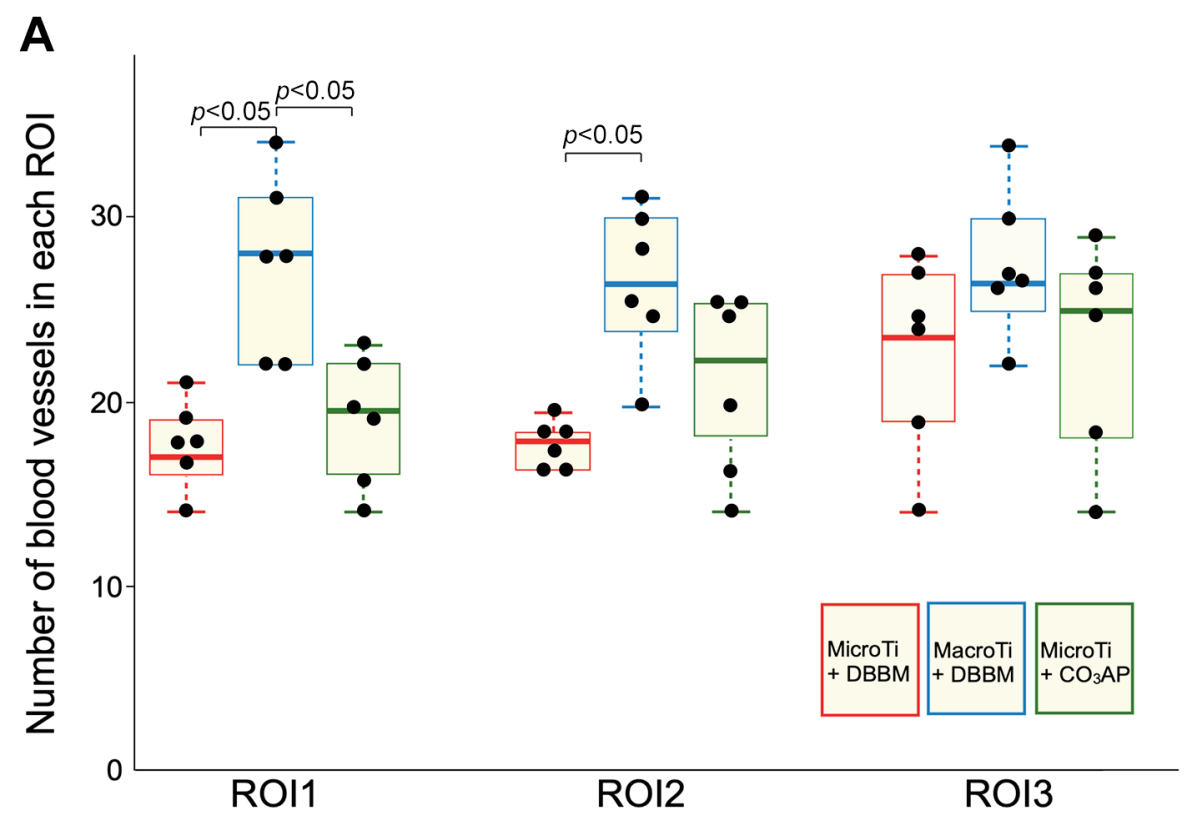

B
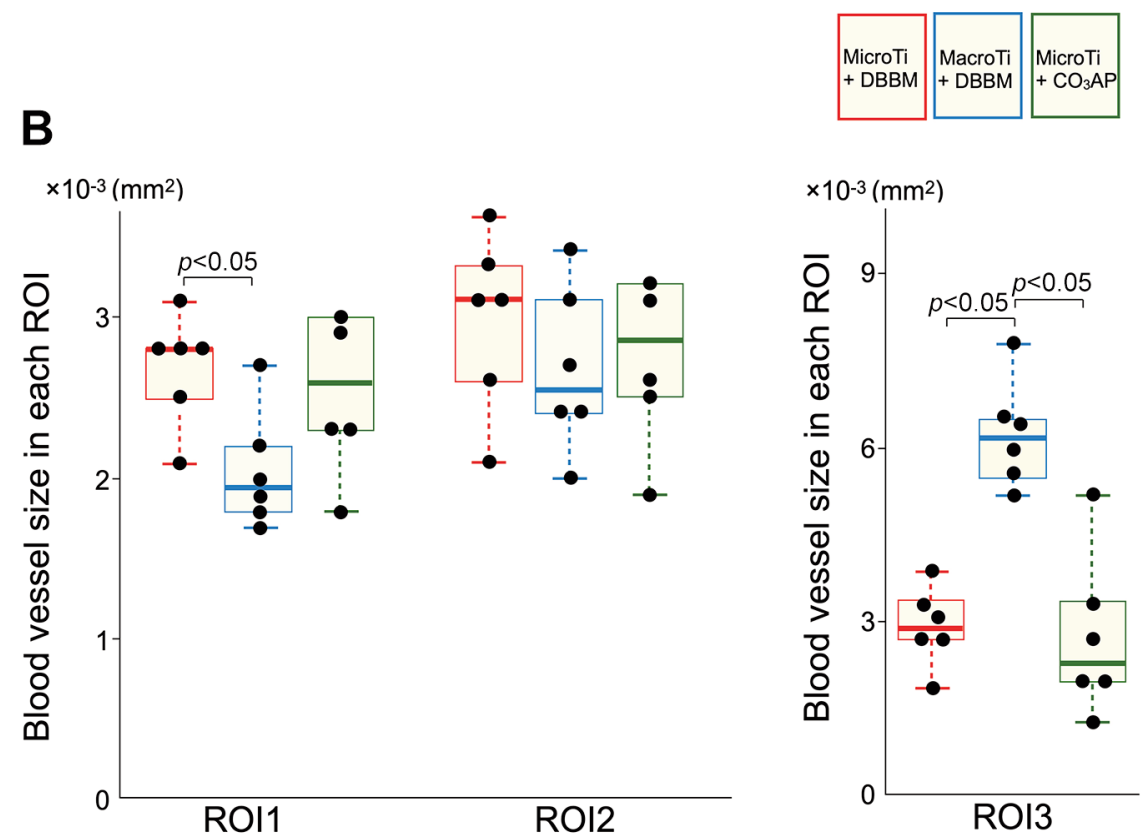

Figure 7. Histomorphometric measurements of angiogenetic parameters after 12 weeks of healing are represented as box plots. Number of blood vessels in each ROI (A); Blood vessel size in each ROI $\left(\mathrm{mm}^{2}\right)(B)$. The median, percentiles, and maximum and minimum values of each group are displayed.

$35.8 \pm 3.7 \%$, MicroTi+CO 3 AP: $34.0 \% \pm 3.8$ ) (Figure 6B). In the lower third of the ROI (ROI1),the numbers of blood vessels was smaller in the microporous titanium groups than in the macroporous titanium group (MicroTi+DBBM: 17.3 \pm 2.4 , Macro Ti +DBBM: 27.5 \pm 4.8 , MicroTi+CO $\left.\mathrm{CO}_{3} \mathrm{AP}: 19.0 \pm 4.6\right)$ (Figure 7A). In the upper third of the ROI (ROI3), there was no significant difference in the number of blood vessels between the three groups (MicroTi+DBBM: 22.5 \pm 5.2 , Macro

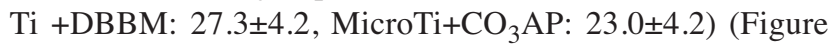
$7 \mathrm{~A})$. In the analysis of blood vessels size in the upper third of ROI, blood vessels were smaller in the microporous titanium groups than in the macroporous titanium group 
(MicroTi+DBBM: $2.9 \pm 0.7 \times 10^{-3} \mathrm{~mm}^{2}$, Macro Ti + DBBM: $6.2 \pm 0.9 \times 10^{-3} \mathrm{~mm}^{2}$, MicroTi+CO ${ }_{3} \mathrm{AP}: 2.8 \pm 1.7 \times 10^{-3} \mathrm{~mm}^{2}$ (Figure 7B).

\section{Discussion}

In this study, we examined the influence of microporous titanium on the vertical GBR. Histological observation confirmed more vigorous bone regeneration in the microporous groups than in the microporous group. In the upper part of the GBR sites, microporous titanium meshes prevented invasion of soft tissue, and enabled microangiogenesis. Our data showed superiority of microporous titanium in angiogenesis and ample blood supply, which play a critical role in promoting bone regeneration.

In micro-CT analysis, a radiopaque image depicts both calcified and mineralized bone substitutes. Thus, differentiating the newly formed bone tissue from transplant particles is impossible. Our results showed a gradual increase in bone volume in all three groups, indicating successful bone regeneration in each group. Histological analysis confirmed vigorous bone regeneration in microporous groups. This indicates that a pore diameter in the micrometer range is more suitable for bone regeneration compared to a pore diameter in the millimeter range.

Wang et al. described four major biological requirements for predictable GBR: primary wound closure, angiogenesis, space maintenance, and stability (19). During GBR surgery, the addition of bone substitutes and mechanical barriers plays a major role in space maintenance. In the healing phase following GBR surgery, the growth of new blood vessels inside the secured space is one of the most important factors for successful GBR. The initial healing step is characterized by the formation of a blood clot. This is followed by the replacement of the blood clot with granulation tissue. Blood vessels are bound to the granulation tissue and are key to the osteoid formation of woven bone. An intimate relationship exists between newly formed blood vessels and de novo bone formation. Potential sources of blood clots and blood vessels communicate with the marrow spaces. Min et al. examined the effect of marrow penetration in rabbit calvarium GBR model titanium caps (20). They showed greater bone augmentation in animals with marrow penetration than in those without penetration. Blood supply from the marrow is a rich source of growth factors and undifferentiated cells.

The periosteal covering the barrier could be another candidate for the origin of blood supply. Considering angiogenesis from the periosteal, the permeability of mechanical barriers is required. Our group compared several sizes of macropores in rat calvarial models that were similar to those used in the present study (6). The pore size created at the lid of the barrier was identical to that of the pores used in commercially available titanium meshes. As a result, barrier permeability in meshes with pores in the millimeter range resulted in less augmented bone, compared with the occlusive barrier. This could be attributed to the fact that fibrous tissue ingrowth occurred from the outside by passing through the millimeter-range pores. The histological results obtained from the macroporous group in the present study correspond with this result. However, the microporous groups showed fibrous tissue volume in the upper part of the cylinders and an abundant volume of newly formed woven bone. In the upper part of the GBR sites just beneath the periosteal tissue, sufficient microvasculature was also observed in the microporous groups. Thus, it could be concluded that micrometer-range pores enabled microangiogenesis and prevent soft tissue ingrowth.

In the present study, two types of particulate bone grafts were placed inside the cylinders covered with microporous titanium meshs: bovine bone xenograft material (DBBM) and synthetic bone substitute $\left(\mathrm{CO}_{3} \mathrm{AP}\right)$. DBBM is a widely utilized bone graft material, especially in countries where the use of allografts is not permitted. Although xenografts were first developed to act as a standard replacement material similar to autografts and allografts, xenografts typically do not resorb over time. $\mathrm{A} \mathrm{CO}_{3} \mathrm{AP}$ bone substitute was recently developed as an alternative to allografts and xenografts (21). Commercially available $\mathrm{CO}_{3} \mathrm{AP}$ particles are chemically pure; they are manufactured by a dissolutionprecipitation reaction in aqueous solution using a calcite block, rather than through a sintering process. In a previous study on rabbit femurs, at 8 weeks, faster bone formation was observed in defects filled with $\mathrm{CO}_{3} \mathrm{AP}$ than in those filled with DBBM (22). In the present study, we also showed the bone regenerative capacity of $\mathrm{CO}_{3} \mathrm{AP}$, which is comparable to that of DBBM. At 12 weeks, both materials remained almost 30\% unabsorbed. Thus, these bone substitutes can be categorized as unabsorbable osteoconductive materials. Differences in particle appearance between these two bone substitutes were confirmed by micro-CT and histology. At 4 weeks, micro$\mathrm{CT}$ confirmed particle aggregation of $\mathrm{CO}_{3} \mathrm{AP}$ particles. Whereas, DBBM particles were scattered inside the cylinders. In histological sections at 12 weeks, bone formation was confirmed around the aggregated particles of $\mathrm{CO}_{3} \mathrm{AP}$, as well as surrounding the scattered particles of DBBM. The difference in particle appearance may influence the quality of newly formed bone.

The major limitation of the present work was that we only focused on the permeability of the titanium mesh as the characteristic difference between two different products. The size of the pores is not the only relevant factor for titanium mesh in osteogenesis. These two products have several 
differences in their properties, such as thickness, smoothness, and toughness. These properties may be confounding factors in bone regeneration. In future studies, the use of identical titanium meshes (except for their permeability) is ideal for further assessment. Furthermore, the collaborative effect of microporosity and surface structure also needs to be examined in future studies (23).

In conclusion, microporous titanium mesh is a useful osteoconductive material for mechanical barrier in VRA, as it effectively supports angiogenesis from both the dura mater and periosteum.

\section{Conflicts of Interest}

The Authors have no conflicts of interest to declare.

\section{Authors' Contributions}

M.S. performed the experiments and data analysis. A.H. conceived and designed the study and wrote the manuscript. T.Y., Y.O., N.W., and M.F. performed the experiments. S.S. analyzed and interpreted the data for the study. All Authors approved the final version of the manuscript to be published.

\section{Acknowledgements}

This work was financially supported by the Dental Research Center, Nihon University School of Dentistry, Tokyo, Japan. This work was also financially supported by JSPS KAKENHI grants JP19K19061 (Grant-in-Aid for Young Scientists) for A.H and JP17K11810 (Grant-in-Aid for Scientific Research (C)) for S.S.

\section{References}

1 Plonka AB, Urban IA and Wang HL: Decision tree for vertical ridge augmentation. Int J Periodontics Restorative Dent 38(2): 269-275, 2018. PMID: 29447321. DOI: $10.11607 /$ prd 3280

2 Dahlin C, Linde A, Gottlow J and Nyman S: Healing of bone defects by guided tissue regeneration. Plast Reconstr Surg 81(5): 672-676, 1988. PMID: 3362985. DOI: 10.1097/00006534198805000-00004

3 Kochi G, Sato S, Fukuyama T, Morita C, Honda K, Arai Y and Ito $\mathrm{K}$ : Analysis on the guided bone augmentation in the rat calvarium using a microfocus computerized tomography analysis. Oral Surg Oral Med Oral Pathol Oral Radiol Endod 107(6): e42-e48, 2009. PMID: 19464642. DOI: 10.1016/ j.tripleo.2009.02.010

4 Oginuma T, Sato S, Udagawa A, Saito Y, Arai Y and Ito K: Autogenous bone with or without hydroxyapatite bone substitute augmentation in rat calvarium within a plastic cap. Oral Surg Oral Med Oral Pathol Oral Radiol 114(5 Suppl): S107-S113, 2012. PMID: 23063386. DOI: 10.1016/j.oooo.2011.08.004

5 Kubota T, Hasuike A, Naito M, Tsunori K, Min S and Sato S: Enhancement of bone augmentation in osteoporotic conditions by the intermittent parathyroid hormone: an animal study in the calvarium of ovariectomized rat. Int J Oral Maxillofac Implants 33(5): 1003-1010, 2018. PMID: 30231085. DOI: 10.11607/ jomi.6326
6 Yamamoto T, Hasuike A, Koshi R, Ozawa Y, Ozaki M, Kubota $\mathrm{T}$ and Sato $\mathrm{S}$ : Influences of mechanical barrier permeability on guided bone augmentation in the rat calvarium. J Oral Sci 60(3): 453-459, 2018. PMID: 30101821. DOI: 10.2334/josnusd.170362

7 Simion M, Fontana F, Rasperini G and Maiorana C: Vertical ridge augmentation by expanded-polytetrafluoroethylene membrane and a combination of intraoral autogenous bone graft and deproteinized anorganic bovine bone (Bio Oss). Clin Oral Implants Res 18(5): 620-629, 2007. PMID: 17877463. DOI: 10.1111/j.1600-0501.2007.01389.x

8 Urban IA, Montero E, Monje A and Sanz-Sánchez I: Effectiveness of vertical ridge augmentation interventions: A systematic review and meta-analysis. J Clin Periodontol 46 Suppl 21: 319-339, 2019. PMID: 30667522. DOI: 10.1111/jcpe. 13061

9 Soldatos NK, Stylianou P, Koidou VP, Angelov N, Yukna R and Romanos GE: Limitations and options using resorbable versus nonresorbable membranes for successful guided bone regeneration. Quintessence Int 48(2): 131-147, 2017. PMID: 27834419. DOI: $10.3290 /$ j.qi.a37133

10 Hämmerle CH, Jung RE, Yaman D and Lang NP: Ridge augmentation by applying bioresorbable membranes and deproteinized bovine bone mineral: a report of twelve consecutive cases. Clin Oral Implants Res 19(1): 19-25, 2008. PMID: 17956571. DOI: 10.1111/j.1600-0501.2007.01407.x

11 Urban IA, Lozada JL, Jovanovic SA, Nagursky H and Nagy K: Vertical ridge augmentation with titanium-reinforced, densePTFE membranes and a combination of particulated autogenous bone and anorganic bovine bone-derived mineral: a prospective case series in 19 patients. Int J Oral Maxillofac Implants 29(1): 185-193, 2014. PMID: 24451870. DOI: 10.11607/jomi.3346

12 von Arx T, Hardt N and Wallkamm B: The TIME technique: a new method for localized alveolar ridge augmentation prior to placement of dental implants. Int J Oral Maxillofac Implants 11(3): 387-394, 1996. PMID: 8752560.

13 Xie Y, Li S, Zhang T, Wang C and Cai X: Titanium mesh for bone augmentation in oral implantology: current application and progress. Int J Oral Sci 12(1): 37, 2020. PMID: 33380722. DOI: $10.1038 / \mathrm{s} 41368-020-00107-\mathrm{Z}$

14 Ikeno M, Hibi H, Kinoshita K, Hattori H and Ueda M: Effects of the permeability of shields with autologous bone grafts on bone augmentation. Int J Oral Maxillofac Implants 28(6): e386e392, 2013. PMID: 24278940. DOI: 10.11607/jomi.te19

15 Jang YS, Moon SH, Nguyen TT, Lee MH, Oh TJ, Han AL and Bae TS: In vivo bone regeneration by differently designed titanium membrane with or without surface treatment: a study in rat calvarial defects. J Tissue Eng 10: 2041731419831466, 2019. PMID: 30834101 . DOI: 10.1177/2041731419831466

16 Hasegawa H, Masui S and Ishihata $\mathrm{H}$ : New microperforated pure titanium membrane created by laser processing for guided regeneration of bone. Br J Oral Maxillofac Surg 56(7): 642643, 2018. PMID: 29954619. DOI: 10.1016/j.bjoms. 2018.05.005

17 Hasegawa H, Masui S, Ishihata H, Kaneko T, Ishida D, Endo M, Kanno C, Yamazaki M, Kitabatake T, Utsunomiya S, Izumi K and Sasaki K: Evaluation of a newly designed microperforated pure titanium membrane for guided bone regeneration. Int J Oral Maxillofac Implants 34(2): 411-422, 2019. PMID: 30883620. DOI: $10.11607 /$ jomi.6777 
18 Ishikawa $\mathrm{T}$ and Ueno $\mathrm{D}$ : Vertical ridge augmentation with a honeycomb structure titanium membrane: a technical note for a 3-dimensional curvature bending method. J Oral Implantol 47(5): 411-419, 2021. PMID: 33027523. DOI: 10.1563/aaid-joiD-20-00262

19 Wang HL and Boyapati L: "PASS" principles for predictable bone regeneration. Implant Dent 15(1): 8-17, 2006. PMID: 16569956. DOI: 10.1097/01.id.0000204762.39826.0f

20 Min S, Sato S, Murai M, Okuno K, Fujisaki Y, Yamada Y and Ito K: Effects of marrow penetration on bone augmentation within a titanium cap in rabbit calvarium. J Periodontol 78(10): 1978-1984, 2007. PMID: 17916000. DOI: 10.1902/jop. 2007.070086

21 Ishikawa K: Bone substitute fabrication based on dissolutionprecipitation reactions. Materials 3(2): 1138-1155, 2018. DOI: $10.3390 / \mathrm{ma} 3021138$
22 Fujisawa K, Akita K, Fukuda N, Kamada K, Kudoh T, Ohe G, Mano T, Tsuru K, Ishikawa K and Miyamoto Y: Compositional and histological comparison of carbonate apatite fabricated by dissolution-precipitation reaction and Bio-Oss ${ }^{\circledR}$. J Mater Sci Mater Med 29(8): 121, 2018. PMID: 30032409. DOI: 10.1007/ s10856-018-6129-2

23 Hartjen P, Hoffmann A, Henningsen A, Barbeck M, Kopp A, Kluwe L, Precht C, Quatela O, Gaudin R, Heiland M, Friedrich RE, Knipfer C, Grubeanu D, Smeets R and Jung O: Plasma electrolytic oxidation of titanium implant surfaces: Microgroovestructures improve cellular adhesion and viability. In Vivo 32(2): 241-247, 2018. PMID: 29475905. DOI: 10.21873/invivo.11230

Received September 17, 2021

Revised October 9, 2021

Accepted October 27, 2021 